\title{
Estimating the Underlying Signal Waveform and Synchronization Jitter from Repeated Measurements
}

\author{
Jesper Martinsson*, (student member), Fredrik Hägglund, (student member), and Johan E. Carlson, (member) \\ EISLAB, Dept. of Computer Science and Electrical Engineering, Luleå University of Technology, SE-971 87 Luleå, Sweden. \\ *Email: Jesper.Martinsson@1tu.se
}

\begin{abstract}
In this paper we present a synchronization technique, for applications using repeated or periodically excited measurements. The problem with existing techniques is their limitations to specific signal and noise conditions, such as white Gaussian noise or narrowband signals. The proposed method extracts statistical information about the underlying signal and noise in the measurements to obtain good synchronization (asymptotically optimal). The Cramér-Rao lower bound (CRLB) is derived for the synchronization problem, including bounds for the underlying signal waveform and the covariance of the noise. The method, which is the maximum-likelihood estimator for both white and colored Gaussian noise, is compared with standard sub-sample estimation and aligning techniques using Monte Carlo simulations. The results show significant improvements compared to standard synchronization techniques.
\end{abstract}

\section{INTRODUCTION}

In many ultrasonic systems, it is possible to perform repeated measurements. Given that the repeated measurements are perfectly synchronized, an estimate of the underlying signal waveform is easily obtained by averaging the measured signals. An estimate of the measurement noise and its statistical properties can then be found by subtracting the average from each measurement.

However, perfect synchronization is often difficult to achieve in practice, due to variations caused by synchronization jitter.

Assume that the retrieved data is a collection of $M$ repeated unsynchronized noisy measurements of an ultrasonic signal. The signal model can be written as

$$
y_{m}[n]=x\left(n \cdot T_{s}-\tau_{m}\right)+w_{m}[n],
$$

for $m=1,2, \cdots, M$ repeated measurements and $n=$ $1,2, \cdots, N$ time samples. Here $y_{m}[n]$ represents the $m$ :th measurement, where $x\left(n \cdot T_{s}\right)$ is the underlying (unknown) signal, assumed to bandlimited below the Nyquist limit, $w_{m}[n]$ the measurement noise, $T_{s}$ is the sampling time, and $\tau_{m}$ the corresponding (unknown) synchronization jitter delay.

Accurate estimates of the measured data's first and second order statistics are important and also required in many applications, for example to: optimally weight objective functions when fitting models or parameters to measured data; obtain uncertainty bounds for estimated models and parameters; detect model errors; and for experimental design [1]. Averaging unsynchronized signals causes a low-pass filtering effect that destroys this information.
The problem of compensating for synchronization jitter is often solved by estimating the sub-sample time delay with respect to one of the received signals, and then pre-aligning the whole set before estimating the underlying signal and the noise covariance. The aligning usually involves interpolation in the time-domain, and the sub-sample time delay estimation often involves some interpolation of the cross-correlation function. Currently available methods assumes either white noise or narrowband signals, see e.g. [2], [3], [4].

In this paper a different procedure is proposed, that does not rely on any of these assumptions. Instead we use the entire set of repeated measurements to simultaneously estimate the underlying signal waveform, the covariance matrix of the noise, and the sub-sample synchronization delays. The estimator is the maximum likelihood estimator (MLE), in the presence of white or colored Gaussian noise, independent of the signal bandwidth, as long as the Nyquist sampling criterion is fulfilled.

The estimator is derived and implemented using a frequency domain approach, which means that classical time-domain interpolation and finite difference approximations of derivatives are avoided.

Section IV then evaluates the performance of the estimator.

\section{THEORY}

In this section, the signal model for the estimation problem is first rewritten in the frequency domain, and then the loglikelihood function, required to form the MLE is stated. The MLE is derived for two cases, one where the covariance matrix of the noise is known a priori, and then for the case where it is not known. Also, an additional iterative procedure for the second case is presented, that reduces the computational complexity and the sensitivity against local maxima.

\section{A. Frequency domain representation}

Transforming the problem into the discrete frequency domain, has a number of advantages compared to standard time domain techniques [5]. In the discrete frequency domain, Eq. (1) can be rewritten as

$$
\tilde{y}_{m}[k]=\exp \left\{-j \omega_{k} \tau_{m}\right\} \tilde{x}[k]+\tilde{w}_{m}[k]
$$

for $k=1,2, \cdots, N$ frequency points, where $\tilde{\mathbf{y}}_{m}=$ $\operatorname{DFT}\left\{\mathbf{y}_{m}\right\}, \tilde{\mathbf{x}}=\operatorname{DFT}\{\mathbf{x}\}, \tilde{\mathbf{w}}_{m}=\operatorname{DFT}\left\{\mathbf{w}_{m}\right\}$, and $\omega_{k}$ is the corresponding angular frequency. 


\section{B. The log-likelihood function}

Assuming $\tilde{\mathbf{w}}_{m} \sim \mathcal{C N}\left(\mathbf{0}, \mathbf{C}_{\tilde{\mathbf{w}}}\right)$ and $\tilde{\mathbf{w}}_{m}$ is independent of $\tilde{\mathbf{w}}_{l}$ for $m \neq l$, the log-likelihood function for $M$ repeated measurements is

$$
\ln (p(\tilde{\mathbf{Y}} ; \tilde{\mathbf{x}}, \boldsymbol{\tau}))=A-\sum_{m=1}^{M} \tilde{\mathbf{e}}_{m}^{H} \mathbf{C}_{\tilde{\mathbf{w}}}^{-1} \tilde{\mathbf{e}}_{m},
$$

where

$$
\begin{aligned}
\tilde{\mathbf{Y}} & =\left[\tilde{\mathbf{y}}_{1}, \cdots, \tilde{\mathbf{y}}_{M}\right], \\
\boldsymbol{\tau} & =\left[\tau_{1}, \cdots, \tau_{M}\right]^{T}, \\
A & =-M\left(N \ln (\pi)+\ln \left(\operatorname{det}\left(\mathbf{C}_{\tilde{\mathbf{w}}}\right)\right)\right), \\
\tilde{\mathbf{e}}_{m} & =\tilde{\mathbf{y}}_{m}-\mathbf{G}\left(\tau_{m}\right) \tilde{\mathbf{x}} \\
\mathbf{G}\left(\tau_{m}\right) & =\operatorname{diag}\left(\left[\exp \left\{-j \omega_{1} \tau_{m}\right\}, \cdots, \exp \left\{-j \omega_{N} \tau_{m}\right\}\right]^{T}\right) .
\end{aligned}
$$

\section{The MLE with known covariance matrix}

Under the assumption that the covariance matrix is known a priori, maximizing Eq. (3) with respect to $\tau$ and $\tilde{\mathbf{x}}$ is a separable nonlinear weighted least squares problem. The problem is linear in $\tilde{\mathbf{x}}$ but nonlinear in the synchronization delays $\tau$. For a given $\tau$ a closed form solution exists for maximizing Eq. (3) with respect to $\tilde{\mathbf{x}}$. Setting the complex gradient,

$$
\frac{\partial \ln (p(\tilde{\mathbf{Y}} ; \tilde{\mathbf{x}}, \boldsymbol{\tau}))}{\partial \tilde{\mathbf{x}}}=\sum_{m=1}^{M}\left(\mathbf{G}^{H}\left(\tau_{m}\right) \mathbf{C}_{\tilde{\mathbf{w}}}^{-1}\left(\tilde{\mathbf{y}}_{m}-\mathbf{G}\left(\tau_{m}\right) \tilde{\mathbf{x}}\right)\right)^{*},
$$

to zero, the maximum is found when

$$
\hat{\tilde{\mathbf{x}}}=\frac{1}{M} \sum_{m=1}^{M} \mathbf{G}^{H}\left(\tau_{m}\right) \tilde{\mathbf{y}}_{m} .
$$

The equality is given by the diagonal structure of $\mathbf{G}\left(\tau_{m}\right)$ and the unitary property $\mathbf{G}^{H}\left(\tau_{m}\right) \mathbf{G}\left(\tau_{m}\right)=\mathbf{I}$. By inserting the closed-form expression for $\hat{\tilde{\mathbf{x}}}$ into Eq. (3), the dimensionality of the non-linear optimization problem is reduced significantly to a maximization of

$$
\ln (p(\tilde{\mathbf{Y}} ; \hat{\tilde{\mathbf{x}}}, \boldsymbol{\tau}))=A-\sum_{m=1}^{M} \tilde{\varepsilon}_{m}^{H} \mathbf{C}_{\tilde{\mathbf{w}}}^{-1} \tilde{\varepsilon}_{m}
$$

with respect to $\tau$ only, where

$$
\tilde{\boldsymbol{\varepsilon}}_{m}=\tilde{\mathbf{y}}_{m}-\mathbf{G}\left(\tau_{m}\right) \hat{\tilde{\mathbf{x}}} .
$$

\section{The MLE with unknown covariance matrix}

The derivations in Section II-C relies on a priori knowledge of the covariance matrix of the noise. If this information is not available, which is often the case, it can be estimated from the repeated measurements.

Since the estimation is done in the frequency domain, we assume that the diagonal properties of the covariance matrix apply [5], i.e. $\mathbf{C}_{\tilde{\mathbf{w}}}=\operatorname{diag}\left(\left[\sigma_{\tilde{w}}^{2}[1], \cdots, \sigma_{\tilde{w}}^{2}[N]\right]^{T}\right)$, the $\log$ likelihood function in Eq. (5) can be simplified

$$
\ln \left(p\left(\tilde{\mathbf{Y}} ; \hat{\tilde{\mathbf{x}}}, \boldsymbol{\sigma}_{\tilde{\mathbf{w}}}^{2}, \boldsymbol{\tau}\right)\right)=-\sum_{m=1}^{M} \sum_{k=1}^{N} \frac{\tilde{\varepsilon}_{m}^{*}[k] \tilde{\varepsilon}_{m}[k]}{\sigma_{\tilde{w}}^{2}[k]}+\sigma_{\tilde{w}}^{2}[k]+\ln (\pi)
$$

with respect to $\sigma_{\tilde{\mathbf{w}}}^{2}$ and $\tau$. However, for a given $\tau$ the minimum variance unbiased estimate (MVUE) [6] of $\sigma_{\tilde{w}}^{2}[k]$ is given by

$$
\hat{\sigma}_{\tilde{w}}^{2}[k]=\frac{1}{M-1} \sum_{m=1}^{M} \tilde{z}_{m}^{*}[k] \tilde{z}_{m}[k],
$$

where $\tilde{z}_{m}[k]=\tilde{\varepsilon}_{m}[k]-M^{-1} \sum_{q=1}^{M} \tilde{\varepsilon}_{q}[k]$. Inserting this closed-form expression into Eq. (7), the dimensionality reduces to the maximization of

$\ln \left(p\left(\tilde{\mathbf{Y}} ; \hat{\tilde{\mathbf{x}}}, \hat{\boldsymbol{\sigma}}_{\tilde{\mathbf{w}}}^{2}, \boldsymbol{\tau}\right)\right)=-\sum_{m=1}^{M} \sum_{k=1}^{N} \frac{\tilde{\varepsilon}_{m}^{*}[k] \tilde{\varepsilon}_{m}[k]}{\hat{\sigma}_{\tilde{w}}^{2}[k]}+\hat{\sigma}_{\tilde{w}}^{2}[k]+\ln (\pi)$

with respect to $\tau$ only.

\section{E. The iterative maximum likelihood estimator (IMLE)}

In noisy measurement situations with few repetitions $M$, the log-likelihood function in Eq. (9) is very sensitive to small changes in the parameters. For these situations, we can resort to sub-optimal methods, where the dependence of the parameter is less nonlinear and the function surface more tractable. A useful approach is to simplify the log-likelihood function so that the dependence of the parameter appears only in the nominator. The idea with the IMLE is to get rid of this dependency by replacing the noise covariance with an estimate obtained from the previous iteration. The IMLE can be summarized as follows:

1) Start by assuming white measurement noise, $\mathbf{C}_{\tilde{\mathbf{w}}}=\sigma_{\tilde{w}}^{2} \mathbf{I}$, and align the measurements by maximizing Eq. (5) with respect to $\tau$.

2) Estimate the covariance matrix, assuming well aligned measurements.

3) Align the measurements by maximizing Eq. (5) with respect to $\boldsymbol{\tau}$, using $\hat{\mathbf{C}}_{\tilde{\mathbf{w}}}$ obtained from step 2 .

4) Iterate from step 2 until convergence.

This method produces a more malleable surface around its global maxima, compared to Eq. (9), at the cost of losing some efficiency. Another advantage is that the full sample covariance matrix can be used (without any complications) if $N$ is small and the asymptotic diagonal properties of $\mathbf{C}_{\tilde{\mathbf{w}}}$ are questionable.

\section{F. Optimization aspects}

Estimating the synchronization jitter results in a nonlinear maximization problem with respect to the parameter $\tau$ and numerical optimization methods must be used to find the maximum. In this paper, the Gauss-Newton method is used to maximize log-likelihood functions.

If the delays $\left|\tau_{m}\right|>T_{s}$, it is recommended to pre-align the measurements to the closest whole sample using standard cross-correlation techniques, to avoid local maxima.

\section{THEORETICAL RESULTS}

In this section the main theoretical results, obtained from the derivations of the estimators and bounds, are discussed and interpreted. 


\section{A. Estimation with singular matrices}

The Fisher information matrix (FIM) [6], given in Appendix A, and the Hessian matrix does not have full column rank, if $\tau \sim \mathbb{R}^{M}$. The reason for this is that for any scalar, $a, \tau$ and $\boldsymbol{\tau}+a \mathbf{1}$ produces the same likelihood. In other words, the Hessian and the FIM, has a null space. This rank deficiency is common in estimation problems with over-parameterized structures [7] and can be solved using different approaches.

In this paper we resolve the rank deficiency by setting $\tau_{k}=$ 0 for some $k$, and make this the reference parameter. This approach eliminates one column and one row in the FIM and Hessian, which then becomes full rank.

\section{Simulation RESUlts}

Four different methods are compared using computer simulations:

SE The "simple" estimator, which is the most commonly used method to synchronize measurements. The measurements are aligned against one of the measured signals (used as reference). Neither the underlying signal waveform nor the covariance is used or estimated.

LSE The least square estimator, which is the MLE in additive white gaussian noise or if the covariance is known a priori, described in Section II-C. The underlying signal waveform is estimated and used.

IMLE The iterative maximum likelihood estimator, described in Section II-E. Both the underlying signal waveform and the covariance are used and estimated.

MLE The maximum likelihood estimator, described in Section II-D. The underlying signal waveform and the covariance are used and estimated.

All methods are implemented in the frequency domain.

\section{A. The simulation signal model}

A Gauss-windowed sinusoid is used to model ultrasonic signals

$$
x(t)=a \exp \left(-t^{2} / b\right) \sin \left(\omega_{0} t\right),
$$

where $a, b$ and $\omega_{0}$ are design parameters. The signal-to-noise ratio (SNR) is then defined as

$$
\mathrm{SNR}=\frac{\sum_{k=1}^{K}|\tilde{x}[k]|^{2}}{\sum_{k=1}^{K} \sigma_{\tilde{w}}^{2}[k]} .
$$

\section{B. The noise model}

The additive noise $w_{m}[n]$ is modeled as Gaussian and colored using discrete ARMA filters as

$$
\tilde{w}_{m}[k]=H[k] \tilde{\epsilon}_{m}[k]+B \tilde{\epsilon}_{m}[k],
$$

where

$$
H[k]=\frac{\sum_{l=1}^{N_{b}} b_{l} \exp (-2 \pi(k-1)(l-1) / N)}{\sum_{l=1}^{N_{a}} a_{l} \exp (-2 \pi(k-1)(l-1) / N)},
$$

is the ARMA filter, $B$ represents a white noise floor, and $\tilde{\epsilon}_{m}[k]$ is the DFT of a white Gaussian noise sequence. This results in a noise covariance calculated as $\sigma_{\tilde{w}}^{2}[k]=|H[k]+B|^{2}$.

\section{Additive colored Gaussian noise}

In Fig. 1(a), the simulated ultrasonic signal is seen together with a realization of a colored noise sequence, using the signal and noise models described above. The signal is modeled as a broadband ultrasonic pulse, with $a=1$, $b=0.005, T_{s}=0.02$ and $\omega_{0} T_{s}=0.4 \pi$. The noise is colored using a third order Chebyshev type I filter with filter coefficients $\mathbf{b}=[0.1059,0.3177,0.3177,0.1059]^{T}, \mathbf{a}=$ $[1.0000,-0.5620,0.7194,-0.3102]^{T}$ and a white noise floor at $B=0.1(-20 \mathrm{~dB})$, and a total SNR of $3 \mathrm{~dB}$.

(a)

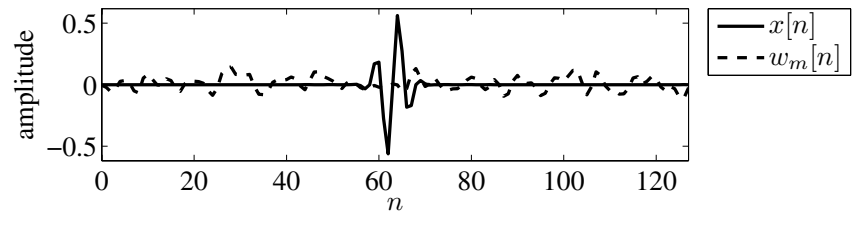

(b)

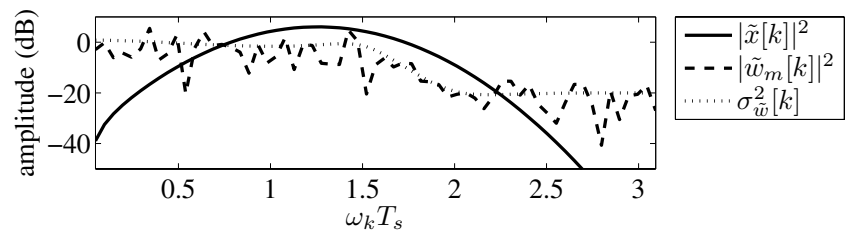

Fig. 1. Signal and noise modeled using Eq. (10) and Eq. (12): (a) discretetime domain representation of the simulated signal $x\left(n \cdot T_{s}\right)$ with $\omega_{0} T_{s}=$ $0.4 \pi$ and a realization of one noise sequence $w_{m}[n]$; (b) discrete-frequency domain representation of the signal, noise and variance.

For the simulations, the synchronization jitter $\tau$ is modeled using independent realizations from a uniform distribution with interval $\left[-T_{s}, T_{s}\right]$, i.e. $\tau_{m} \sim \mathcal{U}\left[-T_{s}, T_{s}\right]$. In Fig. 2(a), the mean square error (MSE) for the different methods can be seen together with the Cramér-Rao lower bound (CRLB), versus the number of measurements $M$, for a constant SNR of $3 \mathrm{~dB}$. For a small number of measurements, the MSEs are comparable between the methods and no significant improvements are observed for the methods using second order statistics. However, as $M$ increases a big improvement can be seen as the IMLE and MLE approaches the CRLB. The difference between SE and LSE is not that big for an SNR of $3 \mathrm{~dB}$. In Fig. 2(b), the MSE versus SNR is shown for $M=10$ measurements. As the SNR drops below $-2 \mathrm{~dB}$, a significant difference is observable between the SE, the LSE. The maximum-likelihood based methods are consistently much better. Note that the CRLB is a lower bound for unbiased estimators, which none of the estimators in this paper are. However, the IMLE and MLE are asymptotically unbiased, which means that the comparison is valid for a sufficiently large data size or high SNR conditions.

\section{Conclusions}

In this paper we derived a maximum likelihood estimator for the synchronization jitter, the underlying signal waveform, and their covariance matrices. All parameters are estimated 
(a)

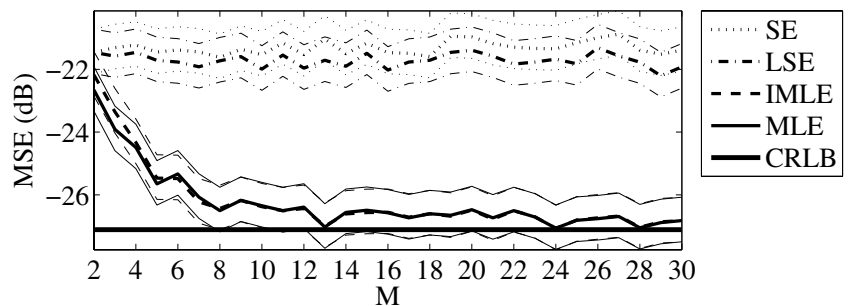

(b)

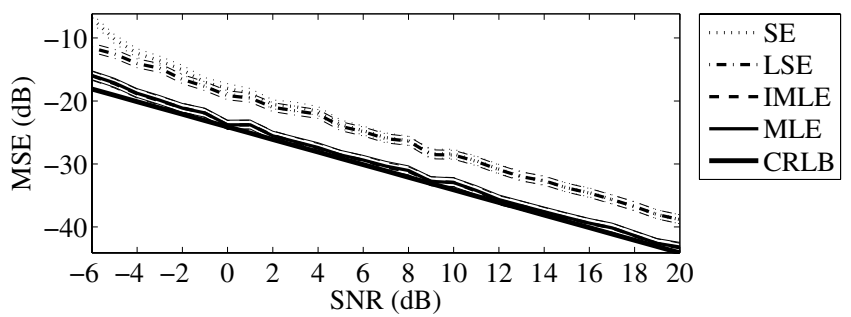

Fig. 2. Mean square error (MSE) comparison of $\hat{\tau}_{m}$ using 500 realizations. The thin lines mark the $99 \%$ confidence intervals. (a) MSE versus the number of measurements $M$, with the noise conditions described in Fig. 1; (b) MSE versus SNR, with $M=10$ and noise colored as in Fig. 1 (except for varying SNR).

simultaneously without any assumptions needed about white noise or narrowband signals.

The method is compared with other sub-sample time delay estimation methods, for wideband signals in the presence of colored noise. We demonstrated with simulations that the variance of the estimates is significantly lower using the proposed method, than for related techniques. The estimator's variances were also compared with the theoretical lower bound (CRLB). As the number of repeated measurements increase the proposed estimator approaches the CRLB.

\section{APPENDIX}

\section{A. Calculation of Cramér-Rao lower bound (CRLB)}

This section briefly outlines the steps of deriving the CRLB of the variance of the estimated parameters.

The Fisher information matrix (FIM) for complex valued signals in complex Gaussian noise can be found in [6]. Applied to the measurements in $\tilde{\mathbf{Y}}$, where $\tilde{\mathbf{y}}_{m} \sim \mathcal{C N}\left(\tilde{\mathbf{s}}_{m}, \operatorname{diag}\left(\boldsymbol{\sigma}_{\tilde{\mathbf{w}}}^{2}\right)\right)$ and $\tilde{\mathbf{w}}_{m}$ is independent of $\tilde{\mathbf{w}}_{l}$ for $m \neq l$, the FIM is given by

$$
\begin{aligned}
{[\mathbf{F}(\boldsymbol{\theta})]_{a, b}=} & \sum_{m=1}^{M} \sum_{k=1}^{K} 2 \Re\left\{\frac{\partial \tilde{s}_{m}^{*}[k]}{\partial \theta_{a}} \frac{1}{\sigma_{\tilde{w}}^{2}[k]} \frac{\partial \tilde{s}_{m}[k]}{\partial \theta_{b}}\right. \\
& \left.+\frac{1}{2 \sigma_{\tilde{w}}^{4}[k]} \frac{\partial \hat{\sigma}_{\tilde{w}}^{2}[k]}{\partial \theta_{a}} \frac{\partial \hat{\sigma}_{\tilde{w}}^{2}[k]}{\partial \theta_{b}}\right\},
\end{aligned}
$$

where

$$
\begin{aligned}
\tilde{s}_{m}[k] & =\exp \left(-j \omega_{k} \tau_{m}\right) \tilde{x}[k], \\
\boldsymbol{\theta} & =\left[\Re\{\tilde{\mathbf{x}}\}^{T}, \Im\{\tilde{\mathbf{x}}\}^{T}, \boldsymbol{\sigma}_{\tilde{\mathbf{w}}}^{2^{T}}, \boldsymbol{\tau}^{T}\right]^{T} .
\end{aligned}
$$

Inserting the derivatives the FIM can be expressed as

$$
\mathbf{F}(\boldsymbol{\theta})=\left[\begin{array}{cccc}
\mathbf{A} & \mathbf{0} & \mathbf{0} & \mathbf{D} \\
\mathbf{0} & \mathbf{A} & \mathbf{0} & \mathbf{E} \\
\mathbf{0} & \mathbf{0} & \mathbf{B} & \mathbf{0} \\
\mathbf{D}^{T} & \mathbf{E}^{T} & \mathbf{0} & \mathbf{C}
\end{array}\right]
$$

where

$$
\begin{aligned}
A_{a, b} & =\delta_{a, b} \frac{2 M}{\sigma_{\tilde{w}}^{2}[a]}, \quad(K \times K) \\
B_{a, b} & =\delta_{a, b} \frac{M}{\sigma_{\tilde{w}}^{4}[a]}, \quad(K \times K) \\
C_{a, b} & =\delta_{a, b} 2 \sum_{k=1}^{K} \frac{\left|j \omega_{k} \tilde{x}[k]\right|^{2}}{\sigma_{\tilde{w}}^{2}[k]}, \quad(M-1 \times M-1) \\
D_{a, b} & =2 \frac{\omega_{a} \Im\{\tilde{x}[a]\}}{\sigma_{\tilde{w}}^{2}[a]}, \quad(K \times M-1) \\
E_{a, b} & =-2 \frac{\omega_{a} \Re\{\tilde{x}[a]\}}{\sigma_{\tilde{w}}^{2}[a]}, \quad(K \times M-1) .
\end{aligned}
$$

The CRLB for an unbiased estimator is then given by the inverse of the FIM [6] as

$$
\operatorname{cov}(\hat{\boldsymbol{\theta}}) \geq \operatorname{CRLB}(\boldsymbol{\theta})=\mathbf{F}^{-1}(\boldsymbol{\theta}) .
$$

Looking only at the time delays, $\tau$, the corresponding CRLB can be shown to be

$$
\operatorname{var}(\hat{\boldsymbol{\tau}}) \geq \mathbf{C R L B}(\boldsymbol{\tau})=\frac{1}{c}(\mathbf{I}+1),
$$

where

$$
c=2 \sum_{k=1}^{K} \frac{\left|j \omega_{k} \tilde{x}[k]\right|^{2}}{\sigma_{\tilde{w}}^{2}[k]} .
$$

\section{REFERENCES}

[1] J. Schoukens and R. Pintelon, "Estimation of nonparametric noise models," in IEEE Instrumentation and Measurement, (Sorrento, Italy), pp. 102-106, 2006.

[2] A. Grennberg and M. Sandell, "Estimation of Subsample Time Delay Differences in Narrowband Ultrasonic Echoes Using the Hilbert Transform Correlation," IEEE Trans. Ultrason., Ferroelec., and Freq. Contr., vol. 41, no. 5, pp. 588-595, 1994.

[3] B. R. Fischer and A. Medvedev, "Laguerre domain estimation of time delays in narrowband ultrasonic echoes," in 14th Triennial IFAC World Congress, (Beijing, China), pp. 361-366, 1999.

[4] S. Björklund and L. Ljung, "A review of time-delay estimation techniques," Tech. Rep. ISSN: 1400-3902, Linköping University, 2004.

[5] R. Pintelton and J. Schoukens, System Identification: A Frequency Domain Approach. IEEE Press, 2001.

[6] S. M. Kay, Fundamentals of Statistical Signal Processing: Estimation Theory, vol. 1. Prentice Hall, 1993.

[7] P. Stoica and T. L. Marzetta, "Parameter estimation problems with singular information matrices," IEEE Trans. Signal Proc., vol. 49, pp. 8790, January 2001. 